\title{
What Counts as a Newtonian System? The View from Norton's Dome
}

\author{
Samuel C. Fletcher*
}

November 30, 2010

\begin{abstract}
If the force on a particle fails to satisfy a Lipschitz condition at a point, it relaxes one of the conditions necessary for a locally unique solution to the particle's equation of motion. I examine the most discussed example of this failure of determinism in classical mechanics - that of Norton's dome - and the range of current objections against it. Finding there are many different conceptions of classical mechanics appropriate and useful for different purposes, I argue that no single conception is preferred. Instead of arguing for or against determinism, I stress the wide variety of pragmatic considerations that, in a specific context, may lead one usefully and legitimately to adopt one conception over another in which determinism may or may not hold.
\end{abstract}

*Thanks to Jeff Barrett, David Malament, Peter Vickers, and Jim Weatherall for helpful comments, and to the audiences of both the Southern California Philosophy of Physics Group and the Ninth Logic, Mathematics, and Physics Graduate Philosophy Conference at the University of Western Ontario for comments on earlier versions. Part of the present work was written with the support of a National Science Foundation Graduate Research Fellowship. 


\section{Introduction}

Classical mechanics is often held as the paragon of a deterministic theory, but there are in fact many ways in which this ideal has been challenged. Some such challenges involve supertasks of denumerable particles interacting and leading to spontaneous self-excitation; others, like the "space invaders" scenario, involve massive particles, traveling under their mutual gravitational influence, appearing from spatial infinity at an arbitrary time $[9,10]$. But perhaps the simplest class of examples, which I focus on in this paper, are systems whose initial conditions do not uniquely fix their equations of motion.

Though seemingly novel in the literature, such examples have been known since at least 1806, when Poisson first investigated dynamical equations of the forms $d v / d t=-a v^{n}$ and $d^{2} x / d t^{2}=a x^{n}$ [26]. These equations have multiple solutions for some initial conditions when $0<n<1$ and $0<|n|<1$, respectively. Poisson, however, did not develop the subject further. In the mid-nineteenth century, Duhamel [7] and Cournot [5] published textbooks that discuss these classes of equations, followed by Boussinesq in the 1870s [3], whose tome was republished through the early 1920s. Curiously, Boussinesq in particular came to see systems governed by these equations as a technical solution to Laplace's demon and as the origin of free will, ${ }^{1}$ but his work seems to have faded from interest as the rise of the quantum theory directed scientists and philosophers away from the classical conception of Laplace's demon [6].

It is then not too surprising that discussion of this class of differential equations was noticeably absent from debates in the mid-twentieth century about the status of determinism in classical mechanics. ${ }^{2}$ In the early 1990s, however, a pair of articles by Hutchison revived cases of the equations studied by Poisson $[12,13]$ to argue against time-reversal invariance in classical mechanics. But the ensuing debate ignored them, perhaps because Hutchison merely described an abstract dynamical system and did not give a physical model for the differential equations involved.

By contrast, the most recent example - that of Norton's dome [23] — uses a concrete and familiar model to illustrate the alleged failure of determinism. ${ }^{3}$ In the example of the dome, a point mass initially at rest on the dome's apex slides down spontaneously at some arbitrary time on account of the dome's special shape. Unlike most commentators, who have argued on various grounds to reject the dome, Malament has brought attention to an underlying

\footnotetext{
${ }^{1}$ He did not, however, investigate how ubiquitous such systems might be.

${ }^{2}$ See Hoering [11] for an overview of the terms of that debate, which focused mostly on issues of predictability.

${ }^{3}$ Norton originally concocted the example as part of an argument against causation as an underlying principle in modern physics, including classical mechanics, but this claim will not play a role in the rest of this paper.
} 
vagueness in the definition of a Newtonian system $[22] .{ }^{4}$ For him, the answer to the question of determinism in classical physics depends on what exactly one takes a Newtonian system to be. Similarly, Wilson has argued that the question of determinism in classical mechanics presupposes a univocal theory of classical mechanics that just is not there, and that no attempt to fashion such a theory can be completely successful [33].

I take these observations as the key to understanding the debate, and argue that the disagreement between Norton and his interlocutors ultimately rests on their differing accounts of the contents of Newtonian theory, which in turn leads to their divergence over what counts as a proper Newtonian system. As soon as one specifies which class of mathematical models one refer to by "classical mechanics," one can unambiguously formulate and perhaps answer the question of determinism as a precise mathematical statement. But, I stress, there is no a priori reason to choose a sole one among these. In practice, the choice of a particular formulation of classical mechanics will depend largely on pragmatic factors like what one is trying to do with the theory. Instead of conceiving classical mechanics as a single canonical theory with a fully delineated subject matter, I leave its content open so that practitioners may decide which formulation best fits their purposes, whatever they may be.

My plan is as follows. I begin in $\S 2.1$ by reviewing how determinism can fail if the forces acting on a dynamical system do not satisfy a certain continuity condition called Lipschitz continuity. Then in $§ 2.2$ I recapitulate the details of Norton's dome, explicating some of its (perhaps unapparent) mathematical features. $\S 3$ details the many criticisms of Norton's dome. In particular: $§ 3.1$ examines claims that Norton has neglected to apply some principle of mechanics; $\S 3.2$ that he has interpreted Newton's laws incorrectly; $\S 3.3$ that the dome should be rejected because it is in some crucial sense "unphysical"; and $\S 3.4$ that the dome employs improper idealizations, where I also introduce in some detail two illustrative examples from electrostatics. In $\S 4$, I review an attempt to transcend objections that aim at features specific to explicit physical models. Throughout I argue that each objection amounts to picking particular contents for the theory, and that the disagreement between Norton and his interlocutors results from the different choices corresponding to their different purposes. As I state in the concluding section ( $\$ 5)$, moreover, I do not wish to advocate for any formalism in particular to decide whether determinism succeeds; rather, I emphasize the pragmatic considerations that guide us in adopting one classical model or other.

\footnotetext{
${ }^{4}$ I will use "classical" and "Newtonian" as synonyms unless otherwise specified.
} 


\section{Building Up the Dome}

\subsection{Setting the Ground}

I will say that a given system is deterministic or satisfies determinism if and only if its equation of motion, whose dynamics are described by a set of differential equations, has a unique solution to its initial value problem. For example, in Newtonian mechanics, the equation of motion $\vec{r}(t)$ for some point particle of mass $m$ satisfies

$$
\vec{F}(\vec{r})=m \frac{d^{2} \vec{r}}{d t^{2}}
$$

where $\vec{F}(\vec{r}): D \rightarrow \mathbb{R}^{3}$ (with $D \subseteq \mathbb{R}^{3}$ ) is the force on the particle. If in addition the equation of motion satisfies some initial conditions, e.g.,

$$
\begin{aligned}
\vec{r}\left(t_{0}\right) & =\vec{r}_{0}, \\
\frac{d \vec{r}}{d t}\left(t_{0}\right) & =\vec{v}_{0},
\end{aligned}
$$

then the Picard-Lindelöf theorem from the theory of ordinary differential equations guarantees that there is a unique maximally extended solution to the equation of motion, provided that the force $\vec{F}$ is locally Lipschitz continuous on its domain $D .{ }^{5}$ Explicitly, I say $F$ is Lipschitz continuous, satisfies a Lipschitz condition, or simply is Lipschitz on its domain $D$ if and only if there is a constant $K>0$ such that $\forall x, y \in D,|F(x)-F(y)| \leq K|x-y|$. So $F$ is locally Lipschitz continuous when, for every $x \in D$, there is a neighborhood $U$ of $x$ such that the restriction of $F$ to $U$ is Lipschitz continuous. Intuitively, Lipschitz continuity prohibits the slope of the tangent line to a curve from getting too large. (See fig. 1.)

Thus, a system consisting of a massive point particle undergoing Newtonian dynamics (eq. (1)) via a locally Lipschitz force is deterministic insofar as the specification of the state of the particle in question - here, its position and velocity - at a particular time $t_{0}$ locally fixes the history of the particle, i.e., its state at each time $t$ in a neighborhood of $t_{0} \cdot{ }^{6}$ But what happens when not all of the above conditions are met? Relaxing the continuity condition so that $F$ is non-Lipschitz at a single point can yield uncountably many solutions to eq. (1)

\footnotetext{
${ }^{5}$ One typically proves this first for first-order ordinary differential equations, then extends it to higherorder equations. See, for example, Arnol'd [1] pgs. 36-38, 104-105.

${ }^{6}$ The locality of this kind of determinism can be highlighted by reference to "space invader" scenarios, in which the specification of the initial conditions of the system in question fixes its history for only a finite period of time $[9,10]$. But this locality as such will not play a role in the examples of this paper.
} 


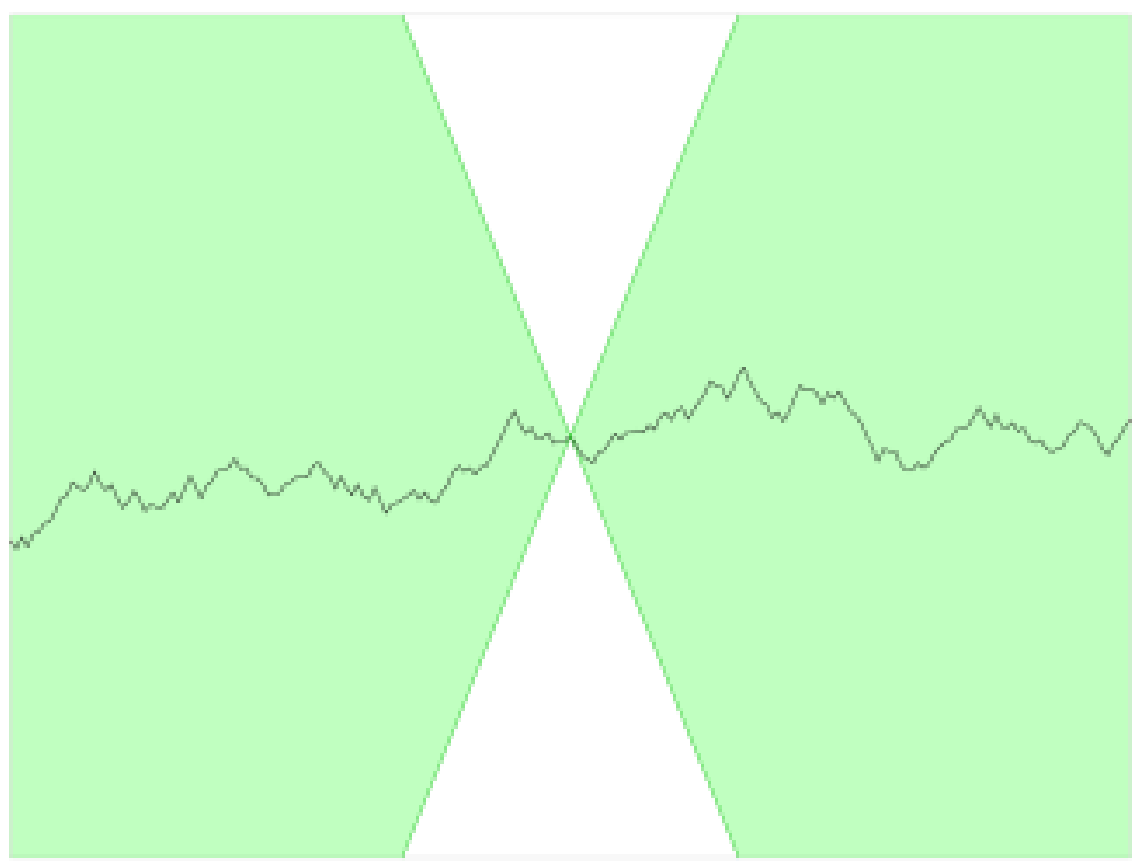

Figure 1: If a function is Lipschitz at a point, the image of the function must lie within the shaded cones whose slopes are determined by the Lipschitz constant $K$.

that satisfy the specified initial conditions. For example, set $\vec{r}_{0}=\vec{v}_{0}=\mathbf{0}$, and take

$$
F(r)=m b^{2} r^{a}
$$

to be a radial force with $0<a<1$ and $b$ a constant whose units depend on the choice of $a$; eq. (4) is non-Lipschitz at the origin. ${ }^{7}$ Then for the radial component of the equation of motion there is both a trivial solution,

$$
r(t)=0
$$

and a nontrivial one:

$$
r(t)=\left(\frac{(1-a)^{2}}{2(1+a)}\right)^{\frac{1}{1-a}}(b[t-T])^{\frac{2}{1-a}} .
$$

Note that $T$ is any time whatsoever. The two solutions given by eqs. $(5,6)$ can be combined in the following way:

$$
r(t)= \begin{cases}0, & \text { if } t \leq T, \\ \left(\frac{(1-a)^{2}}{2(1+a)}\right)^{\frac{1}{1-a}}(b[t-T])^{\frac{2}{1-a}}, & \text { if } t>T .\end{cases}
$$

\footnotetext{
${ }^{7}$ To see why, suppose otherwise; then there must be some constant $K>0$ satisfying $|F(r)| \leq K|r|$. But any choice of $r$ satisfying $0<r<\left(m b^{2} / K\right)^{1-a}$ yields a contradiction.
} 


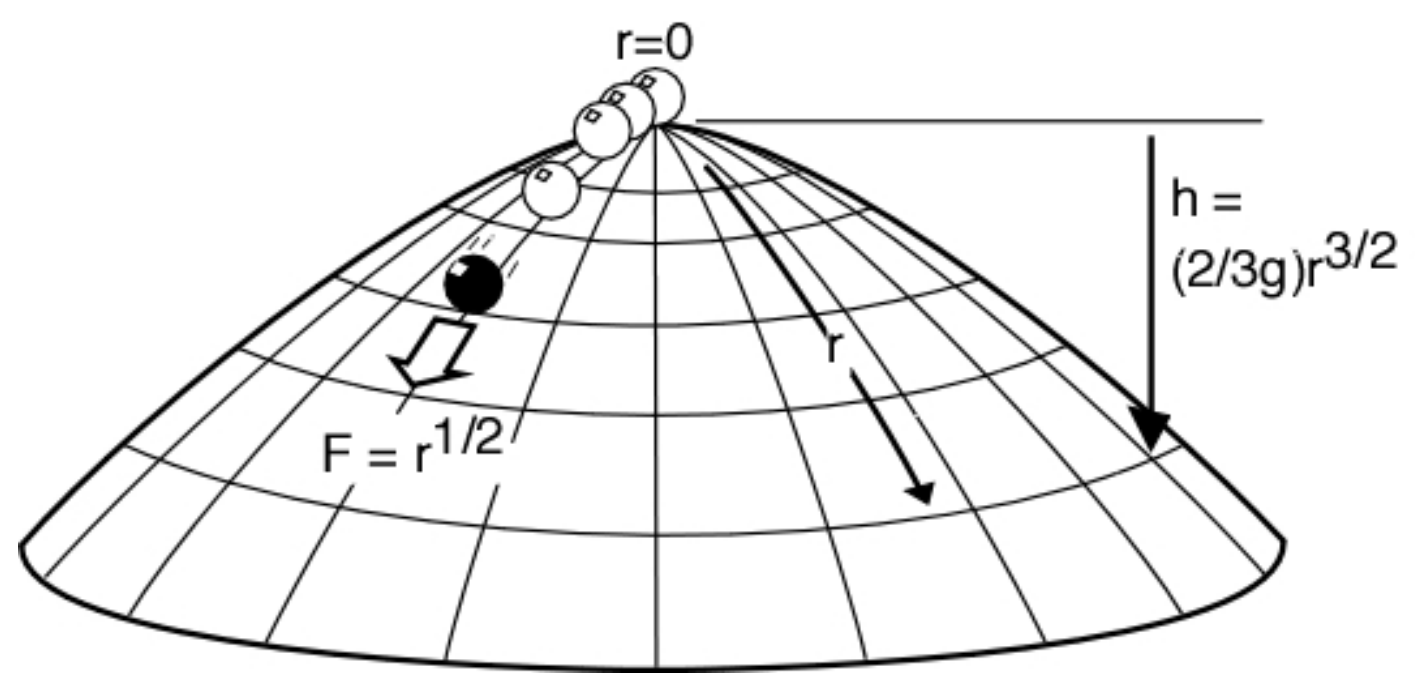

Figure 2: A cartoon of Norton's dome with a unit mass from [23], not to scale, and with the constant $b$ set to unity in the appropriate units.

Thus, according to eq. (7), the point particle sits at the origin until the arbitrary time $t=T$, the last time at which it is at rest; afterward, it moves away as a power of the time elapsed from $t=T$. Because the theory provides no additional information about what the parameter $T$ should be, there are nondenumerably many possible solutions, each corresponding to a possible value of $T \in \mathbb{R}$, and determinism fails. I call this kind of failure of determinism Lipschitz indeterminism, and systems that display it Lipschitz indeterministic.

\section{$2.2 \quad$ Norton's Dome}

The case that Norton considers in [23, 25] is a cylindrically symmetric dome (see fig. 2) on which a point particle of mass $m$ slides under the influence of a uniform gravitational field. The shape of the dome acts as a kind of constraint surface that yields the necessary force equation for Lipschitz indeterminism. For, suppose the particle is constrained to move on the surface, and the equation for the loss of height $h$ from the top of the dome, as a function of the distance traversed along the surface $r$, is given by

$$
h(r)=\left(2 b^{2} / 3 g\right) r^{3 / 2},
$$

with $g$ being the acceleration due to gravity and $b$ a constant. Then the force propelling the particle down the slope is the component of the total force tangential to the surface:

$$
F_{\|}=m g \sin \theta=m g \frac{d h}{d r}=m b^{2} \sqrt{r}
$$


where $\theta$ is the angle between the radial tangent to the surface at the particle's location and the the horizontal. Placing the point particle at rest on the apex of the dome yields the Lipschitz indeterminism outlined in $\S 2.1$ if one picks $a=1 / 2$ in (7), to simplifying the equation of motion to

$$
r(t)= \begin{cases}0, & \text { if } t \leq T \\ \frac{1}{144}(b[t-T])^{4}, & \text { if } t>T\end{cases}
$$

and the force equation (4) to

$$
F(r)=m b^{2} \sqrt{r}
$$

In the case of the dome, the particle at the apex may fall down after any time, and in any of the radial directions. The apex of the dome is special because it is the only location at which the radial force acting on the particle is non-Lipschitz. Not only is this initial position special in this respect, but the zero-magnitude initial velocity is as well. Malament has shown that for any arbitrarily small initial velocity in the radial direction, the point particle loses touch with the surface of the dome, and there is no Lipschitz indeterminism [22]. Gravity is the force pushing the particle into the dome, which, pushing back, constrains it to that surface. But for any non-zero initial velocity, the parabolic arc traced by the particle in free fall extends past the dome; the dome falls away from the particle faster than the particle falls under gravity, for any non-zero initial velocity.

Finally, Norton emphasizes that classical mechanics gives no guidance as to any possible probability distribution over the possible allowed equations of motion. ${ }^{8}$ Even relying on symmetry to assign a uniform probability will not work since there is no such distribution for the parameter $T$, which can take on any real value. One might be tempted instead to assign a probability distribution in analogy with radioactive decay, where the rate of "decay" of the particle is uniform over time. But since such processes require a time constant to define exactly what this rate is, there still remains a parameter totally unspecified by the theory.

\section{Attempts to Demolish the Dome}

Norton [25] considers a variety of objections to his example, classifying them as such: ${ }^{9}$

1. The dome incorrectly or incompletely applies Newtonian mechanics.

\footnotetext{
${ }^{8}$ Although it makes little difference for what follows, I think it would be a mistake even to consider interpreting Lipschitz indeterminism as a species of probabilistic evolution akin, say, to classical stochastic systems without explicitly augmenting the mathematical structure of classical mechanics. Treating fluid flows whose velocity fields can be non-Lipschitz, E and Vanden-Eijnden do just that [8].

${ }^{9}$ We need not view this classification as a partition of possible objections, since some particular objection may include aspects from more than one category. Nevertheless, we can still use them pragmatically to organize possible objections.
} 
2. The system consisting of the dome and point particle is in some crucial sense unphysical:

(a) as overdescription, i.e., as a gauge quantity;

(b) as contradictory, i.e., as contradicting other generally well-established claims of the theory;

(c) as false, i.e., as empirically falsified;

(d) as underdescription, in the sense of it indicating the theory's incompleteness.

3. The analysis of the dome uses improper idealizations.

Many of Norton's responses to these categories of objection are cogent, and I address them in turn along with the relevant criticism from the literature. In each case I argue that these criticisms simply assume a formulation of classical mechanics different from Norton's.

\subsection{Incomplete Application of Newtonian Theory?}

Regarding the first category of objections - that the principles of Newtonian mechanics have been incompletely applied-Norton admits that there may be an added constraint or a modification to an accepted rule of the theory that will disqualify his dome as a legitimate system. For example, by enforcing that all equations of motion be smooth, one can rule out the class of solutions given by (6), ensuring that the unique static solution is given by (5). ${ }^{10}$

But such a rule unduly restricts the set of systems amenable to analysis. One simple example of this is that of a ball rolling off a horizontal table. The net force on the ball changes discontinuously as it loses contact with the table. Korolev initially took this as one approach to throwing out the dome, arguing not for smoothness, but for (the weaker) Lipschitz continuity as an implicit assumption of classical mechanics [17]. Earman has objected that this seems more of an ad hoc addition than smoothness, since one advances it only to rule out Lipschitz indeterminism explicitly [10]. It is not the kind of condition that seems natural to enforce outside of otherwise pathological examples, and for Earman, taking the dome as pathological merely begs the question for determinism. Moreover, as Norton points out, Lipschitz continuity is a sufficient but not necessary condition for the uniqueness of solutions to the equations of motion. In fact, the force on the ball rolling off the table is not Lipschitz at the point where it loses contact, and surely that example is benign. Another important example arises in fluid dynamics with non-Lipschitz velocity fields. For such situations, if Lipschitz indeterminism arises, one can introduce generalized flows, which associate a probability measure with the space of all solutions to the fluid's

\footnotetext{
${ }^{10} \mathrm{~A}$ function $f: \mathbb{R} \rightarrow \mathbb{R}^{n}$ is smooth when its derivatives $\frac{d^{n} f}{d t^{n}}$ exist and are continuous for each $n \in \mathbb{N}$.
} 
equations of motion. ${ }^{11}$ The proper treatment of these flows is an active field of research, one whose practitioners would be loathe to abandon on account of Norton's dome.

One cannot rule out Lipschitz indeterminism tout court, enforcing some kind of continuity across all applications of classical mechanics without in the same stroke eliminating otherwise unproblematic examples. After all, there are innocuous cases like balls rolling off tables and fascinating cases like non-Lipschitz velocity fields in turbulent fluids that resist such a requirement. Yet, in some applications, requiring Lipschitz continuity, say, might make sense depending on the goals of one's research. There are, of course, many ways to do this. That is, even if one were to impose an additional constraint like Lipschitz continuity, there will always be a certain degree of arbitrary stipulation regarding how one wishes to enforce it. Here is a short list that makes no pretension to comprehensiveness:

1. Eliminate any system where at least one trajectory in phase space is Lipschitz indeterministic.

2. Given some system, remove any trajectories in phase space that are Lipschitz indeterministic.

3. Given some trajectory of a system in phase space, remove any points that result in Lipschitz indeterminism.

Depending on one's purposes, one of these options might make more sense than another. For example, the first option rules out Norton's dome as a valid system entirely, even when one does not consider the system's particle initially at rest at the apex. The second disallows one from considering (only) incomplete trajectories along the surface of the dome, while allowing trajectories that come arbitrarily close. The third option is the most austere, removing only a single point of phase space. The first option might be useful if one is interested in classifying deterministic systems, say, but not if one is interested in the dynamics of the system away from the non-Lipschitz point, in which case the third option might be more appealing. Meanwhile, the second option stands attractively for those interested in studying flows under a variety of force laws. Each of these remains an option as a criterion that one can impose fruitfully depending on one's purposes. In a similar manner, there are many ways that one can enforce the Lipschitz condition if one desires by restricting a model's domain of application, or by changing the dynamics by hand at the appropriate places. Eliminating elements of phase space and inserting new dynamics by hand at these points do not constitute new techniques for classical mechanics, and are no more ad hoc than the

\footnotetext{
${ }^{11}$ Such examples can arise naturally in problems of turbulence, since the Navier-Stokes equation in three dimensions is non-Lipschitz in the limit of zero viscosity. See, for example, E and Vanden-Eijnden [8]. (Ironically, Korolev [16] was the first to point this out.)
} 
same techniques used to model, according to one's purposes, classical systems that do not violate determinism.

\subsection{Incorrect Application of Newtonian Theory?}

Zinkernagel has recently argued not that Norton has neglected a tacit assumption of Newtonian mechanics, but that he has incorrectly applied Newton's first law, that "Every body continues in its state of rest, or of uniform motion in a right line, unless it is compelled to change that state by forces impressed upon it" [35]. When properly understood, he claims, the particle beginning at rest at the apex of the dome remains there at rest for all time. Similarly, in the "time-reversal" case where the particle begins on the inclined surface of the dome with just the right initial velocity to bring it to rest at the apex, the particle remains at the apex once it arrives. ${ }^{12}$ Unlike particles on more conventionally shaped domes, the particle sliding up Norton's dome actually does come to rest, at least for a moment. Zinkernagel argues that the particle stays there indefinitely instead of undergoing any kind of indeterministic evolution.

Zinkernagel's proposal has intuitive appeal: particles at rest on conventional domes stay put, and his answer assimilates Norton's dome to these cases. It is also not without historical merit, for it is the same conclusion drawn by Poisson in his landmark study: ${ }^{13}$

However, it is clear from the nature of the problem that if the initial velocity was assumed to be zero, the body should remain at rest. ... If instead the initial velocity is not assumed to be zero, ... [t] he movement of the body at the beginning of time $t$ will be therefore given by the integral [solution]; but when the time becomes equal to $[T]$, the velocity given by this integral will be zero; at the end of a time marked by $[T]$ the body stops moving; its velocity, after that instant, will always be zero, and consequently will no longer be given by the integral [solution], but rather by the particular solution $v=0$.

Thus, from $t=0$ up to $[t=T]$, the motion of the body is represented by the integral [solution], and beyond this instant, it is the particular solution $v=0$ which gives the motion, or, better said, which makes it clear that the body then stops moving. ${ }^{14}$

([26], p. 101)

\footnotetext{
${ }^{12}$ The "time-reversal" here is that of the equations of motion, rather than of the laws of Newtonian mechanics. But really one does not even need that since it is easy to reverse-engineer the appropriate choice of initial velocity to bring the particle to rest at the apex given an initial location on the surface of the dome.

${ }^{13}$ In the quoted passage Poisson is speaking directly about solutions to the differential equation $d v / d t=$ $-a \sqrt{v}$, but in his later remarks on $d^{2} x / d t^{2}=a x^{n}$, of which Norton's dome is a special case, he stresses the same conclusions more briefly.

14 "Or, il est visible par la nature du problème que si la vîtesse initiale était supposée nulle, le corps devrait
} 
Here $T$ is the time at which the particle reaches the apex in the "time-reversal" case, and the integral solution is the non-static solution to the equations of motion.

Of course, Poisson said little to justify his choice of solution beyond asserting that there is no initial force to perturb the particle from its state: "for it is clear that the moving body must remain at its starting point, since at this point its velocity, and the force to which it is subjected, are equal to zero" ([26], p. 104). ${ }^{15}$ That a body will not move unless it undergoes a first cause seems, in fact, to be a significant part of Zinkernagel's argument as well, which hinges on an analysis of "continues" in the first law. He reads "continues" as meaning that every change must have a first cause, so that a particle with uniform velocity at time $t=T$ must remain at that velocity continuously until at least time $t=T+\epsilon$, for some $\epsilon>0$. This criterion selects the particle-at-rest trajectory for Norton's dome, since it requires the net force acting on any particle to be non-zero only on closed intervals or half-open intervals of the form $[a, b)$.

Effectively, Zinkernagel requires that every force have a first cause, an instant at which it "turns on." He reads "uniform" motion, at some time $t$, to mean that there is some $\epsilon>0$ such that the particle has constant velocity on $[t-\epsilon, t+\epsilon]$. Thus, at $t=T$, the particle on the dome is not in a state of rest because $t=T$ is the last instant at which it has zero velocity. But this must mean a force must have acted upon it, for at each point of time $t<T$ the particle was at rest. However, no such force is available. Therefore the particle must stay at rest.

At first this seems to be a puzzling response: surely there are innumerable innocuous Newtonian systems where forces turn on smoothly from zero to non-zero magnitude, so that there is no first instant when the force causes a change. In a note added in proof, Zinkernagel acknowledges this difficulty, but claims that he can circumvent it by considering any such continuously varying force as the limit of a series of discrete forces with first instances, viz. first causes, which he considers to be more fundamental for Newton. He claims that forces that are turned on smoothly can be captured in this framework, while Lipschitz indeterminism cannot.

rester en repos. ...Si, au contraire, la vîtesse initiale n'est pas supposée nulle, [l]e mouvement du corps à l'origine du temps $t$ sera donc représenté par l'intégrale $v=\left[\sqrt{b}-\frac{a t}{2}\right]^{2}$; mais quand le temps sera devenu égal à $\frac{2 \sqrt{b}}{a}$, la vîtesse donnée par cette intégrale sera zéro donc au bout d'un temps marqué par $\frac{2 \sqrt{b}}{a}$ le corps cessera de se mouvoir; sa vitesse, à partir de cet instant, sera toujours zéro, et par consequent elle ne sera plus donnée par l'intégrale $v=\left[\sqrt{b}-\frac{a t}{2}\right]^{2}$, mais bien par la solution particulière $v=0$.

Ainsi, depuis $t=0$ jusqu'à $t=\frac{2 \sqrt{b}}{a}$, le mouvement du corps est représenté par l'intégrale $v=\left[\sqrt{b}-\frac{a t}{2}\right]^{2}$, et au delà de cet instant, c'est la solution particulière $v=0$ qui donne le mouvement, ou pour mieux dire, qui fait voir que le corps cesse alors de se mouvoir." All translations are original.

15 "car il est visible que le mobile doit rester au point de départ, puisqu'en ce point sa vitesse et la force à laquelle il est soumis, sont égales à zéro." 
Essentially, the method advocated in Zinkernagel's note is Euler's method for approximating the solutions to differential equations. There is a sense in which he is right: the difference equations (rather than differential equations) of Euler's method are not indeterministic. But this answer invites other problems. It is well-known that Lipschitz discontinuous functions can behave erratically under Euler methods, the calculated trajectory of a particle moving under a non-Lipschitz force depending significantly on choices that should not matter, like the size of the time step used for the iteration of the difference equations. ${ }^{16}$ In other words, while Lipschitz indeterminism is avoided, one must know ahead of time what the intended trajectory should look like in order to pick a time step that will lead to it.

However, another straightforward objection to Zinkernagel's reading arises through a small modification of Norton's dome. Take the dome and elongate it along the $x$-axis, say, so that it becomes a ridge whose profile in the $y z$-plane remains the same as that of the dome. Next raise the comparative heights of these cross-sections so that the cusp of the ridge becomes a smoothly varying curve - a parabola, say, with a minimum at $x=0$. The resulting ridge resembles a saddle shape, with the special property that each cross-section in the $y z$-plane remains the same as that of the dome (except, perhaps, at the ends). Now consider a particle beginning at rest somewhere on but not at the center of the ridge (i.e., with $x \neq 0$ ). Under the constant force of gravity it will undergo periodic motion back and forth along the ridge, until some time $t=T$ when it will fall down one side or other in the $y$-direction. The particle is never in uniform motion and so Newton's first law does not apply to it, undermining Zinkernagel's objection that Lipschitz indeterminism can be ruled out by considering a proper reading of the first law alone.

Perhaps one can reply to this example by advancing a reading of the first law as applying not to total velocity but to each component of velocity. For in the ridge example above, the $y$ component of the particle's velocity remains zero until the particle falls down spontaneously. At face, the textual evidence for this interpretation seems wanting, but I will not press the case here, for it is clear at this point that Zinkernagel is not arguing about the modern conception of classical mechanics, as Norton is, but about Newtonian mechanics as it was practiced by Newton. Zinkernagel's concern with the subtleties of Newton's formulation of his laws, their implications for the crucial definition of inertial frames, the primacy of discrete forces over continuous ones, and the notions of time and causality they collectively engender all show that he and Norton simply have different subjects of analysis. ${ }^{17} 18$ Perhaps

\footnotetext{
${ }^{16}$ For a philosophical discussion of these issues, see Wilson [32], p. 216-7.

${ }^{17}$ To be fair, in a draft of his paper [24], Norton does consider objections based on interpretations of Newton's laws, but concludes that such debates are independent of his concerns and ultimately omits the discussion in the final version [25].

${ }^{18}$ Cf. Vickers's account of a recent debate on the consistency of classical electrodynamics, in which he
} 
Zinkernagel's analysis bears on whether Newtonian mechanics was deterministic or not. ${ }^{19}$ Given the quotations above, it would be interesting to examine Poisson's reasoning in more detail, and Newton's as well. But that investigation frames a particular formulation of classical mechanics different from Norton's, and is not my concern here.

\subsection{The Dome as Unphysical?}

Norton also has replies to objections that the dome is somehow unphysical. Drawing on the classification given at the beginning of $\S 3$, it is clear that the dome example does not seem to involve overdescription-Lipschitz indeterminism arises from the multiplicity of solutions to the equations of motion, after all - and the only contradictory claim that it could be seen as making is that it fails determinism. ${ }^{20}$ But assuming determinism as an assumption of classical physics is to beg the question, Norton argues, for that is precisely the issue under consideration.

However, in analogy with necessitating, in some way or another, Lipschitz continuity for forces in Newtonian systems as a legitimate and practical constraint in some circumstances, there may be good reasons according to one's purposes to require Newtonian systems to be deterministic simpliciter. For example, Landau and Lifshitz write of a classical mechanical system that "If all the co-ordinates and velocities are simultaneously specified, it is known from experience that the state of the system is completely determined and that its subsequent motion can, in principle, be calculated" ([19], p. 1). Physicists like Landau and Lifshitz are interested in the formalism of mechanics insofar as it can be used to model, predict, and explain empirical phenomena. Because the phenomena they are interested in appear to act deterministically, they are interested only in mathematically deterministic classical systems as described by the theory. They do not take the theory to allow for indeterminism and restrict its application, but rather choose a deterministic formalism explicitly because it fits their purposes best.

The two other construals of "unphysical" here perhaps provide stronger objections. For example, one might claim that the solutions given by eqs. (5) and (6) constitute

argues the parties involved have largely been talking past each other [29]. Unlike Vickers, however, for the purposes of this paper I remain agnostic about whether Norton's formulation of classical mechanics is interesting or important. Recently Vickers has synthesized the morals from both debates to urge discussants not to talk past each other on account of having different conceptions of some theory [31].

${ }^{19} \mathrm{Cf}$. Vickers's discussion of the consistency of Newtonian cosmology prior to mathematical and conceptual developments of the late nineteenth and early twentieth centuries. [30]

${ }^{20}$ Norton repeatedly asserts that Newtonian mechanics is a false theory, so even if it produces a claim contradicting either another claim internal to the theory or a piece of empirical evidence, one should not be surprised; he is interested in the structure of the theory independent of such considerations. Though it may be cold comfort, one can - unlike the "space invaders" examples - construct Lipschitz indeterministic systems within the context of special relativity, a theory certainly "closer" to the truth than classical mechanics. 
underdescription - that only one of the solutions they pick out is correct. If the determined mechanist can rule out all solutions but one, she would reestablish determinism for the dome. A difficulty arises, however, explaining which solutions are the unphysical ones without assuming determinism. One might think that Newton's first law - that a body undergoes acceleration only when acted upon by an outside force - suggests that the particle should remain at rest at the apex of the dome, as Zinkernagel and Poisson advocated, for example. Our intuitions about everyday objects (for what they are worth) verify this: we do not expect objects at rest to spontaneously fall into motion. On the other hand, consider elastic collisions of two point particles in at least two spatial dimensions. Conservation of energy and momentum underdetermine the scattering angle of the particles, which can be put in by hand. Alternately one can assume that the particles reverse their courses in the center-of-mass frame, or decide to use rigid spheres instead of point particles. Whichever method one finds best to use genuinely depends on the circumstances one is trying to model, and a similar moral could be drawn for Norton's dome.

Perhaps one could appeal to empirical evidence as the arbiter - that is, dismiss the solution that fits the data the worst - but because Norton's dome has never been built and tested, there is no such evidence to speak of. In fact, there may never be any evidence that one could produce that bears on Norton's dome. Even if one could construct it perfectly, there is only a single point in phase space - that is, a single position and velocity pair - that exhibits Lipschitz indeterminism. Setting up the system would be practically impossible. Norton responds that this is irrelevant precisely because his interest is in the theory itself:

The dome is not intended to represent a real physical system. The dome is purely an idealization within Newtonian theory. On our best understanding of the world, there can be no such system. For an essential part of the setup is to locate the mass exactly at the apex of the dome and exactly at rest. Quantum mechanics assures us that cannot be done. What the dome illustrates is indeterminism within Newtonian theory in an idealized system that we do not expect to be realized in the world.

([25] p. 793)

This is a striking declaration. Norton is explicit that he is ultimately concerned with the mathematical structure of Newtonian mechanics rather than the theory as it applies to the world.

I would affirm that interest in the formal mathematical structure of some particular conception of Newtonian mechanics is just as legitimate as interest in any of the many other aspects of the theory. But it is not the interest many others have in the theory, because for them the formal theory matters only insofar as it applies to the world. Arnol'd writes of a 
process in time that "the form of the differential equation of the process, and also the very fact of determinacy ... can be established only by experiment, and consequently only with limited accuracy" [1] (p. 14). He then emphasizes that his mathematical treatment of such processes proceed "as if they coincided exactly with our idealized mathematical models" (ibid.). Thus for practitioners of mathematical physics like Arnol'd, the mathematics are an idealized tool whose applicability to any situation of interest is constrained by one's ability to verify, to some sufficient level, the degree of coincidence between the model and the process of interest.

When confronted with the dome, such practitioners are therefore typically unmoved. For example, Kosyakov considers at least two kinds of facts to be major barriers to considering cases of Lipschitz indeterminism compelling in general [15]: first, the forces involved, when posited ab initio, are physically unmotivated, and second, for all known examples, the initial conditions supporting Lipschitz indeterminism are of measure zero. These consideration are exactly what drive Kosyakov to consider electrodynamic examples in one temporal and one spatial dimension, where head-on trajectories are the norm, to instantiate Lipschitz indeterminism. He takes much care to show how Lipschitz indeterminism might be physically motivated, even in his toy model. Because Norton has provided a physical model built from familiar forces and constraints, he overcomes Kosyakov's first hurdle. However the dome remains unobjectionable but unimpressive for those for whom the second - that there are virtually no initial conditions yielding indeterminism - is a significant impediment. ${ }^{21}$

By analogy, consider Landau and Lifshitz's description of the use of point particles in classical mechanics:

One of the fundamental concepts of mechanics is that of a particle. By this we mean a body whose dimensions may be neglected in describing its motion. The possibility of doing so depends, of course, on the conditions of the problem concerned. For example, the planets may be regarded as particles in considering their motion about the Sun, but not in considering their rotation about their axes.

([19], p. 1, italics original)

On this view, one should take seriously the mathematical structures of classical mechanics only insofar as they bear on relevant problems. Furthermore, which problems are relevant depend on one's interests and purposes. The gross astronomy of the solar system can sustain celestial objects of myriad sizes as material points, but finer structures like asteroid belts

\footnotetext{
${ }^{21}$ One might consider a force function $f$ not Lipschitz continuous on some open subset $A$ of its domain. But then $f$ is not continuously differentiable simpliciter on $A$ (see [1], p. 273). One can extend this result to show that in fact $f$ cannot be differentiable on $A$, for the points of continuity of any derivative must be dense and $G_{\delta}$ in $A$, excluding the empty set (see theorem 2.2 of [2].)
} 
and Saturn's rings require elaboration beyond what single points can provide.

From an entirely different and more traditionally philosophical perspective, Lange [20] has argued that for a world truly governed by Newtonian laws, Lipschitz indeterminism is impossible. His thesis is that fundamental physical laws, if they are indeed fundamental, must ipso facto be complete. If this thesis is correct, the system described by Norton's dome - or any other Lipschitz indeterministic system, for that matter - is an instance of incompleteness, since either the path of the particle is underdetermined by the theory, or it is genuinely random, but the theory is mute about the associated probabilities. Thus a world governed in a lawlike manner by a formulation of classical mechanics that allows for Lipschitz indeterminism would be impossible. Someone who is both committed to Lange's completeness thesis and interested in the properties of possible worlds would likely rule out examples of Lipschitz indeterminism, or not take them seriously because they either refer to impossible worlds or are constructed in an explicitly incomplete theory without serious metaphysical implications.

For many who use classical mechanics, then, the physical theory is interesting and relevant only insofar as it serves one's purposes, and I considered above two such purposes ultimately at odds with Norton's conclusion: making testable predictions and drawing conclusions about the metaphysics of possible worlds. For the latter, one might be interested in a world governed lawfully by Newtonian physics, but such a world seems either impossible or incompletely described by those laws. For the former, the instability of particular features like Lipschitz indeterminism may be sufficient evidence that those features, even if predicted by the theory, are not meaningful. Of course, a detailed account of this will need to argue what this instability amounts to, and such an argument will likely need to tread through idealization.

\subsection{Improper Idealization(s)?}

Norton admits that there are many idealizations employed by the dome, including the use of point masses, frictionless sliding, precise initial conditions, infinite curvature at the apex, and complete rigidity in the dome [25]. Korolev has pointed out, in particular, that if the dome were not completely rigid, then it would deform sufficiently to prevent Lipschitz indeterminism [17]. He further constructs other examples of Lipschitz indeterminism using either rigid or elastic bodies, finding that they only work when one assumes the ropes or rods involved are either completely elastic or completely rigid. Thus he concludes that infinite idealizations like these are responsible for Lipschitz indeterminism in general [18].

Norton responds that virtually all of the idealizations he uses are used elsewhere in 
classical mechanics without complaint, and Malament concurs [25, 22]: the mere idealizations themselves cannot be responsible for Lipschitz indeterminism, but rather the particular use of specific idealizations in contexts like the dome. Korolev would then owe Norton an account of what distinguishes the dome from unproblematic cases, but it seems the only relevant feature at hand is the failure of determinism. And to take this as the feature distinguishing cases like Norton's dome is either to beg the question or to repeat the objections of the previous section, which hinge on one's purposes for using classical mechanics rather than on idealization per se.

Moreover, there are additional, simple examples that do not use the same kinds of idealizations that the dome does. For example, consider a spherically symmetric charge distribution that vanishes beyond some finite radius $R$ :

$$
\rho(r)= \begin{cases}\frac{5 c \epsilon_{0}}{2 \sqrt{r}}, & \text { if } 0<r \leq R, \\ 0, & \text { if } r=0 \text { or } r>R .\end{cases}
$$

Here $c$ is a dimensional constant and $\epsilon_{0}$ is the permittivity of free space, as usual. ${ }^{22}$ Note that while $\lim _{r \rightarrow 0^{+}} \rho(r) \rightarrow \infty$, the total charge in the distribution is finite:

$$
\begin{aligned}
Q & =\int_{0}^{R} \int_{0}^{\pi} \int_{0}^{2 \pi} \rho\left(r^{\prime}\right)\left(r^{\prime}\right)^{2} \sin \theta^{\prime} d \phi^{\prime} d \theta^{\prime} d r^{\prime} \\
& =10 \pi c \epsilon_{0} \int_{0}^{R}\left(r^{\prime}\right)^{3 / 2} d r^{\prime} \\
& =4 \pi c \epsilon_{0} R^{5 / 2}
\end{aligned}
$$

On account of the distribution's spherical symmetry, we can use Gauss's Law to find the radial (and only nonvanishing) component of the electric field for when $r \leq R$ :

$$
\begin{aligned}
E_{r}(r) & =\frac{1}{4 \pi \epsilon_{0} r^{2}} \int_{0}^{r} \int_{0}^{\pi} \int_{0}^{2 \pi} \rho\left(r^{\prime}\right)\left(r^{\prime}\right)^{2} \sin \theta^{\prime} d \phi^{\prime} d \theta^{\prime} d r^{\prime} \\
& =\frac{5 c}{2 r^{2}} \int_{0}^{r}\left(r^{\prime}\right)^{3 / 2} d r^{\prime} \\
& =c \sqrt{r}
\end{aligned}
$$

This is precisely the form of the force law needed to replicate the behavior from Norton's

\footnotetext{
${ }^{22}$ Norton found this particular example independently [25] and mentions it briefly. Zimba [34] developed a somewhat similar electrostatic example using an ideal quadrupole and a spherical charge distribution, except with a pair of conical sections symmetric about the $z$-axis removed. He made this construction to avoid any objections regarding "collisions" between the charge distribution and the point particle - an objection, as I outline below, I think is weak - but as a result the force on the point particle is proportional to $\sqrt{|z|}$ only to first order. Thus Zimba's example is not clearly one of Lipschitz indeterminism.
} 
dome. For one can now consider a test particle of charge $q$, which experiences a radial Coulomb force

$$
F_{r}(r)=q E_{r}(r)=q c \sqrt{r}
$$

when $r \leq R$ and, when placed at the origin, its equation of motion is Lipschitz indeterministic in the same way as the dome.

One obvious objection is that the point particle would immediately collide with the charge distribution as soon as it began to move. But while Newtonian mechanics does purport to describe the physics of rigid bodies, it by no means limited to describing them. Though one can do so, it is not required that charge distributions also impose constraints on the motions of particles. This is not as unusual as it seems; historically, the Thomson "plum pudding" model of the atom involved a similar kind of charge distribution with a point particle (or several point particles) within. And in galactic astrophysics, it is quite common to consider massive dust that acts like a fluid, with particles passing through it, modeling the distribution of stars or galaxies as such. Closer to home, a classic textbook problem in freshman mechanics is to consider a point particle dropped through a straight "hole" drilled through the center of the Earth - where the scare quotes indicate that the fact that the hole itself, as an absence of mass, is always ignored, not requiring any special treatment. ${ }^{23}$ Also, the rigidity objection only proscribes a failure of determinism when the sphere is perfectly rigid; for if it is allowed to be deformed in the slightest by the test charge, then that test charge would have already instantiated a failure of determinism by moving to cause the deformation. $^{24}$

Perhaps a stronger objection is that the charge distribution manifests a pathology, since $\rho \rightarrow \infty$ as $r \rightarrow 0$, while $\rho(0)=0$. However, both divergent limits and discontinuous charge distributions show up commonly in previous accepted and unproblematic examples of electrostatics, so they cannot by themselves be cause for rejection. First of all, there is a sense that point particles, whose charge distribution is given by the Dirac distribution, already manifest both of these supposed pathologies. But beyond that, any considerations of charged (one-dimensional) lines, (two-dimensional) shells or (three-dimensional) perfect conductors will involve discontinuous charge distributions, the former two because of their dimensionality and the latter because the net charge on a perfect conductor will distribute itself on the conductor's two-dimensional boundary.

There are also many other physical systems in electrostatics in which the theory states

${ }^{23}$ The particle then undergoes simple harmonic motion. See, for example [27].

${ }^{24} \mathrm{~A}$ related objection that can be handled similarly is accounting for breaking radiation by the point particle. While for the purposes of the example I would take the point particle to be a test charge whose contributions to the electromagnetic fields are to be neglected, allowing for braking radiation does not undermine the example as long as the particle begins moving at all. 


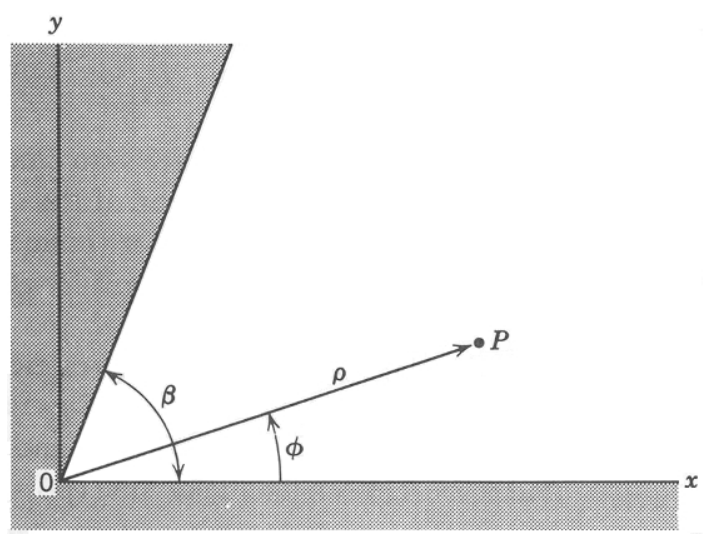

Figure 3: Cross-sectional view of the intersection of two conducting planes at an angle $0<\beta \leq 2 \pi$; if we imagine these planes to be the sides of a conducting box, then for $\beta<\pi$ they constitutes an "inside" corner and for $\beta>\pi$, an "outside" corner of a convex box. [14]

that the charge density diverges. For example, consider a charge $Q$ placed on a perfectly conducting circular disk of radius $a$ centered at the origin. The charge will distribute itself on the disk according to the surface density

$$
\sigma(r)=\frac{Q}{2 \pi a \sqrt{a^{2}-r^{2}}}
$$

for $r<a[14] .{ }^{25}$ Here the charge density diverges at the edge of the disk.

Another example involves the charge density at the corner of a grounded conducting box formed by the intersection of two planes at an angle $0<\beta \leq 2 \pi$, as in fig. 3. Symmetry provides that the charge distribution on each plane must be equal; for each plane, the surface charge density as a function of the distance $r$ from the corner is

$$
\sigma(r)=-\sum_{m=1}^{\infty} \frac{m a_{m}}{4 \beta} r^{(m \pi / \beta)-1} \approx-\frac{a_{1}}{4 \beta} r^{(\pi / \beta)-1}
$$

where the rightmost equation exhibits the dominant behavior near $r=0$, and each $a_{m}$ is a constant determined by the electric potential far away from the corner [14]. Notice that for $\beta>\pi$, which represents the cases for the "outside" corner of a box, the charge density diverges at the corner.

But there are further examples from electrostatics that avoid this possible of divergence issue as well. Consider a system whose setup is analogous to the first except the form of the spherically symmetric charge distribution is different, and the point charge is replaced by an

\footnotetext{
${ }^{25}$ An uncannily careful reader might observe that, to be precise, the derivation of this distribution involves taking a disk whose thickness is negligible compared with its radius; then half of eq. (16) gives the charge distribution on each side.
} 
ideal dipole. ${ }^{26}$ So let the charge density be given by

$$
\rho(r)= \begin{cases}c \epsilon_{0} \sqrt{r} & \text { if } r \leq R \\ 0 & \text { if } r>R\end{cases}
$$

The electric field, by symmetry, is nonvanishing only in its radial component. For $r \leq R$, then,

$$
\begin{aligned}
E_{r}(r) & =\frac{1}{4 \pi \epsilon_{0} r^{2}} \int \rho d V \\
& =\frac{c}{r^{2}} \int_{0}^{r}\left(r^{\prime}\right)^{5 / 2} d r^{\prime} \\
& =(2 c / 7) r^{3 / 2}
\end{aligned}
$$

Now we introduce the ideal dipole with moment $\vec{p}$. Because of the spherically symmetric distribution, the force on the dipole at radius $r$ is given by

$$
\begin{aligned}
\vec{F}(r) & =(\vec{p} \cdot \vec{\nabla}) \vec{E} \\
& =\frac{p_{r}}{r^{2}} \frac{\partial}{\partial r}\left(r^{2} E_{r}(r)\right) \hat{r} \\
& =\frac{2 c p_{r}}{7 r^{2}} \frac{\partial}{\partial r}\left(r^{7 / 2}\right) \hat{r} \\
& =c p_{r} \sqrt{r} \hat{r}
\end{aligned}
$$

where $p_{r}$ is the radial component of the dipole moment at radius $r$ and $\hat{r}$ is the unit vector in the radial direction. There must be some direction in which $p_{r}=\sqrt{p_{x}^{2}+p_{y}^{2}+p_{z}^{2}}$ is nonvanishing for each $r$, for otherwise one would not have a dipole in the first place. After placing the dipole at the origin, determinism fails for these directions - any not orthogonal to $\vec{p}$.

While this example may still be targeted by the rigidity objections that aimed at the original charged sphere, this new charge distribution does not have any of the continuity features that could have been pathological in the previous one. There are no "infinite" idealizations, properly speaking. Instead, the most dubious feature is the ideal dipole. But it is a mathematically legitimate construction, arguably not any more of an idealization than a point particle. Again, this idealization cannot ipso facto warrant rejection. One can only do so on grounds that distinguish it from the harmless multitude. Like in the case of

\footnotetext{
${ }^{26}$ An ideal dipole is a formed as follows. Take two point charges $q$ and $-q$, and let $\vec{d}$ be the displacement vector from $-q$ to $q$. Define the dipole moment as $\vec{p}=q \vec{d}$, then take the limit as $q \rightarrow \infty$ and $\|\vec{d}\| \rightarrow 0$ while holding $\vec{p}$ constant. Note that $\vec{p} \neq 0$, for then otherwise $q=0$.
} 
rigidity for the dome, the only way to do so without begging the question is to fall back on considerations independent of idealization. As Landau and Lifshitz emphasized above, an idealization is only proper or improper with respect to a particular purpose. Like the case of the dome, neither that of the point charge nor that of the ideal dipole can be constructed in a laboratory, and for similar reasons: any imperfection in the distribution or misalignment of the particle's initial conditions ruin the chances for Lipschitz indeterminism. ${ }^{27}$ Whether that disqualifies such systems from being of interest will depend on one's purposes.

\section{Indeterminism Without a Physical Model?}

Another option on Norton's part is to posit explicitly a force like eq. (11) so as to avoid the complications of systems of constraints, deforming continua, and the like. In particular, the example he considers in [25] involves a fundamental length within which the force acts:

$$
F(r)= \begin{cases}m \sqrt{L-r} & \text { if } r \leq L \\ 0 & \text { if } r>L\end{cases}
$$

This is an attractive force between a fixed (i.e., stationary) source at the origin and a massive test particle positioned at rest at a distance $L$ from the source. Shorn of any physically complicating aspects, the only remaining candidate responsible for the failure of determinism is the fact, as before stated, that the force is non-Lipschitz at $r=L$.

One might object, however, to the outright positing of force laws of an atypical form, especially to derive such unexpected and controversial results. Laraudogoitia, for example, is of this opinion:

By introducing more or less exotic force functions, a trivial advantage can be taken of the fact that not every differential equation possesses a unique, global solution, to obtain simple examples of indeterminist [sic] evolution (see, for instance, [12]). This type of result is no more complex than the typical textbook exercise but it is, unfortunately, the least interesting of its kind. Much more relevant are situations in which the only interactions permitted are those specific to Newtonian mechanics, i.e. gravitation and/or collisions ...

The idea seems to be that, if one is going to derive broad metaphysical conclusions from a theory, one must use the "fundamental" forces or interaction of that theory, not merely ones that can be treated theoretically. Callender, in defending the time-reversal invariance

\footnotetext{
${ }^{27}$ There is an additional issue with the ideal dipole, as any dipole with non-zero physical size will not suffice for Lipschitz indeterminism.
} 
of classical mechanics against dissipative forces, uses a similar argument: because one has good reason to believe that, at bottom, all forces are conservative, it is very likely that dissipative forces are merely phenomenological [4]. If one could conveniently keep track of every molecular collision, then one would not need a separate treatment of them within the theory. One should therefore only be interested in actual forces that arise from the actual ontology of the theory. And certainly, gravitation, particle scattering, rigid bodies, and electrostatics are all good candidates for classically fundamental interactions, while the force of $(21)$ is not. ${ }^{28}$

But on the other hand, Wilson questions whether or not one can consistently divide classical mechanics into fundamental and phenomenal forces [33]. Instead, he argues that what is commonly referred to as "classical mechanics" is actually at least three separate sets of tools that often give mutually incompatible interpretations of the same issue: point particle mechanics, the mechanics of rigid bodies and perfect constraints, and continuum mechanics. ${ }^{29}$ The fundamental forces in one may be only approximate and phenomenological in another. Each of them has descriptive gaps that reach all the way to their cores, so while appeal to one from another can locally give a way to paper over those gaps, such repairs cannot be effected globally. Wilson uses the metaphor of a stool with six uneven legs: as it gets pushed around, applied to model some system or other, it might settle onto some set of legs or switch as the emphasis is changed even slightly, but it cannot settle onto all of them. Therefore there is no unitary "classical mechanics" to speak of, only a motley collection of formalisms that are useful for certain purposes, and no global conclusions about them all together can be drawn from just one.

One point of disagreement I have with Wilson is that he seems to suggest the indeterminism of Norton's Dome arises from the decomposition of forces into "reactive" - those that constrain the particle to the surface - and "active" - those that perform work on the particle. Roberts has argued, for example, that this analysis is neither necessary nor sufficient for indeterminism [28]. (While the failure of sufficiency is obvious, the electrostatic examples above show that it is also not necessary.) Then the force decomposition is rather just an artifact of one's particular formulation of classical mechanics, while the Lipschitz condition is responsible for Lipschitz indeterminism in all cases. Despite this difference, Wilson's position is very similar to my own, as I have described it: there is a plurality of choices

\footnotetext{
${ }^{28}$ This is perhaps why, idealization notwithstanding, Norton's dome is intuitively so much more compelling than (21) and Hutchison's idealized forces $[12,13]$.

${ }^{29}$ Wilson acknowledges that "they split into further subdivisions as further questions are pressed" ([33] p. 176) so that, depending on how fine-grained one would like to be, there may be many more kinds of classical mechanics than just three. I agree that there is a plurality of formulations of classical mechanics but am skeptical that there really are neat subdivisions, even at the coarsest level. The elaboration of this worry, however, is a topic for another time.
} 
one can make about the formulation of the theory and the idealizations proper within it, and that the choice of a particular version of classical mechanics will depend upon one's purposes and interests. Therefore one cannot draw conclusions across each and every version. I would hasten to add, however, that one can draw conclusions about determinism within a particular formalism of classical mechanics pinned down with enough mathematical rigor, but that one cannot employ this strategy globally since some formulations may be incompatible with each other, e.g., requiring only smooth forces or not.

\section{Conclusions}

In the foregoing sections I have illustrated several examples of Lipschitz indeterminism, including Norton's dome, a saddle-shape ridge, two examples from electrostatics involving a point test charge and an ideal dipole, respectively, and an abstract force without a familiar physical model. Innumerably many more, of course, could be produced. I have also recapitulated and critiqued many of the attempts in the literature to argue that these examples are illegitimate. Some such objections focus on features specific to the models involved, asserting that they are in some way unphysical or use illicit idealizations. Few, if any, of these features, however, are shared by all these models. Broader objections came from more general principles, either focusing on the proper treatment of Newton's laws themselves or on properties that constructed models must have in order to be interesting or remarkable.

My argumentative strategy throughout has often been to show that each kind objection seeking to rule out Lipschitz indeterminism altogether is too strong, in the sense that it also rules out as illegitimate examples of systems that are otherwise unproblematic. A determined objector, however, could still stand behind one of these objections if she is ready to throw out those cases as well. She might claim that new developments in physics can easily move us to reevaluate the cogency of previously innocuous cases, and Norton's dome is just one such example.

But such an objector owes an explanation for why these systems seemed so innocent and why they heretofore caused no trouble, especially since the cases thereby spoiled are so common. Even if such an explanation is forthcoming, there is still the predicament of how to treat these cases in an inoffensive way. In practice, the old methods would continue, because for the purposes of those cases no additional complications are needed. For various intentions and goals, I have tried to argue, one picks the version of classical mechanics with the assumptions most suited for accomplishing them. The historian of physics may limn the practices of Newton and his contemporaries, the mathematician may consider only forces with certain continuity properties, and the physicist may focus on properties of models that 
can be investigated through experiment.

What of the philosopher, meanwhile? She can still answer the question of determinism, but only within the context of a precisely specified formulation of the theory that may not be the same as that of working physicists or even other philosophers. For example, she may be interested in answering under what conditions of motion and charge distribution electromagnetic forces are non-Lipschitz, or whether or not there is a configuration of matter in Newtonian gravitation that leads to Lipschitz indeterminism. But she does not have a single privileged theory that rises above the rest and from which she can make unqualified claims about the whole of classical mechanics. There is a plurality of different conceptions of classical mechanics, any particular choice of which is not a priori privileged over any other, since each is chosen to be useful in its own way. Consequently one may impose continuity conditions on forces according to one's purposes, restricting the domain of application of a model, or modifying its behavior, according to the pragmatic concerns of the questions at hand.

\section{References}

[1] Arnol'd, Vladimir I. Ordinary Differential Equations. Trans. Roger Cooke. Berlin: Springer-Verlag, 1992.

[2] Bruckner, Andrew M. Differentiation of Real Functions. Lecture Notes in Mathematics 659. Eds. A. Dold and B. Eckmann. Berlin: Springer-Verlag, 1978.

[3] Boussinesq, Joseph. Théorie Analytique de la Chaleur, Mise en Harmonie avec la Thermodynamique et avec la Théorie Mécanique de la Lumière. Compléments au Tome III: Conciliation du Véritable Déterminisme avec l'Éxistence de la Vie et de la Liberté Morale. Paris: Gauthier-Villars, 1878, 1879 (2nd ed.), 1922 (3rd ed.).

[4] Callender, Craig. "The Metaphysics of Time Reversal: Hutchison on Classical Mechanics," British Journal for the Philosophy of Science 46 (1995): 331-340.

[5] Cournot, Antoine Augustin. Traité élémentaire de la théorie des fonctions et du calcul infinitésimal. Paris: L. Hachette, 1841, 1857 (2nd ed.).

[6] Deakin, Michael A. B. "Nineteenth Century Anticipations of Modern Theory of Dynamical Systems," Archive for History of the Exact Sciences 39.2 (1988): 183-194.

[7] Duhamel, Jean Marie Constant. Cours de mécanique. Paris: Mallet-Bachelier, 1845, 1853 (2nd ed.), 1862 (3rd ed.). 
[8] E, Weinan, and Eric Vanden-Eijnden. "A note on generalized flows," Physica D 183 (2003): 159-174.

[9] Earman, John. "Aspects of Determinism in Modern Physics," Philosophy of Physics. Ed. Jeremy Butterfield and John Earman. Handbooks of the Philosophy of Science. Ed. D. Gabbay, P. Thagard and J. Woods. Amsterdam: Elsevier, 2007.

[10] Earman, John. "How Determinism Can Fail in Classical Physics and How Quantum Physics Can (Sometimes) Provide a Cure," Philosophy of Science 75 (2008): 817-829.

[11] Hoering, Walter. "Indeterminism in Classical Physics," British Journal for the Philosophy of Science 20 (1969): 247-255.

[12] Hutchison, Keith. "Is Classical Mechanics Really Time-reversible and Deterministic?" British Journal for the Philosophy of Science 44 (1993): 307-323.

[13] Hutchison, Keith. "Temporal Asymmetry in Classical Mechanics," British Journal for the Philosophy of Science 46 (1995): 219-234.

[14] Jackson, John David. Classical Electrodynamics. 2nd ed. New York: Wiley, 1975.

[15] Kosyakov, B. P. "Is Classical Reality Completely Deterministic?" Foundations of Physics 38 (2008): 76-88.

[16] Korolev, Alexandre V. "Indeterminism, Asymptotic Reasoning, and Time Irreversibility in Classical Physics," Phil. of Sci. Assoc. 20th Biennial Mtg. (Vancouver): PSA 2006 Contributed Papers, http://philsci-archive.pitt.edu/archive/00003003/01/IndeterminismPSA2006.pdf.

[17] Korolev, Alexandre V. "Indeterminism, Asymptotic Reasoning, and Time Irreversibility in Classical Physics," Philosophy of Science 74 (2007): 943-956.

[18] Korolev, Alexandre V. "The Norton-Type Lipschitz-Indeterministic Systems and Elastic Phenomena: Indeterminism as an Artefact of Infinite Idealizations," Philosophy of Science (2010): forthcoming; Phil. of Sci. Assoc. 21st Biennial Mtg (Pittsburgh, PA): PSA 2008 Contributed Papers.

[19] Landau, L. D. and E. M. Lifshitz. Mechanics. Course of Theoretical Physics, vol. 1. 3rd ed. Trans. J. S. Sykes and J. S. Bell. Oxford: Elsevier, 1976.

[20] Lange, Marc. "Must the Fundamental Laws of Physics be Complete?" Philosophy and Phenomenological Research 78.2 (2009): 312-345. 
[21] Laraudogoitia, Jon Perez. "On indeterminism in classical dynamics," European Journal of Physics 18 (1997): 180-181.

[22] Malament, David B. "Norton's Slippery Slope." Philosophy of Science 75 (2008): 799816.

[23] Norton, John D. "Causation as Folk Science." Philosophers Imprint 3.4 (2003), http://www.philosophersimprint.org/003004/; reprinted in Causation, Physics, and the Constitution of Reality: Russell's Republic Revisited. Ed. Huw Price and Richard Corry. New York: Oxford UP, 2007.

[24] Norton, John D. "The Dome: An Unexpectedly Simple Failure of Determinism," Phil. of Sci. Assoc. 20th Biennial Mtg. (Vancouver): PSA 2006 Symposia. http: //www.pitt.edu/ jdnorton/papers/DomePSA2006.pdf.

[25] Norton, John D. "The Dome: An Unexpectedly Simple Failure of Determinism," Philosophy of Science 75 (2008): 786-98.

[26] Poisson, Siméon Denis. "Mémoire Sur les Solutions particulières des Équations différentielles et des Équations aux différences," Journal de l'École Polytechnique 6.13 (1806): 60-125.

[27] Resnick, R., D. Halliday and K. S. Krane. Physics. Vol. 1. 4th ed. New York: Wiley, 1992.

[28] Roberts, Bryan W. "Wilson's Case Against the Dome: Not Necessary, Not Sufficient." (Unpublished manuscript, Feb. 2009.) http://www.pitt.edu/〜bwr6/research/RobertsB_WilsonDiscussion.pdf

[29] Vickers, Peter. "Frisch, Muller, and Belot on an Inconsistency in Classical Electrodynamics," British Journal for the Philosophy of Science 59 (2008): 767-792.

[30] Vickers, Peter. "Was Newtonian cosmology really inconsistent?" Studies in History and Philosophy of Modern Physics 40 (2009): 197-208.

[31] Vickers, Peter. "Theory-Eliminativism as a Methodological Tool," forthcoming manuscript.

[32] Wilson, Mark. Wandering Significance. New York: Oxford UP, 2006.

[33] Wilson, Mark. "Determinism and the Mystery of the Missing Physics," British Journal for the Philosophy of Science 60 (2009): 173-193. 
[34] Zimba, Jason. "Inertia and Determinism," British Journal for the Philosophy of Science 59 (2008): 417-428.

[35] Zinkernagel, Henrik. "Causal Fundamentalism in Physics," EPSA Philosophical Issues in the Sciences: Launch of the European Philosophy of Science Association. Ed. Mauricio Suárez, Mauro Dorato, and Miklós Rédei. Dordrecht: Springer, 2010. 